
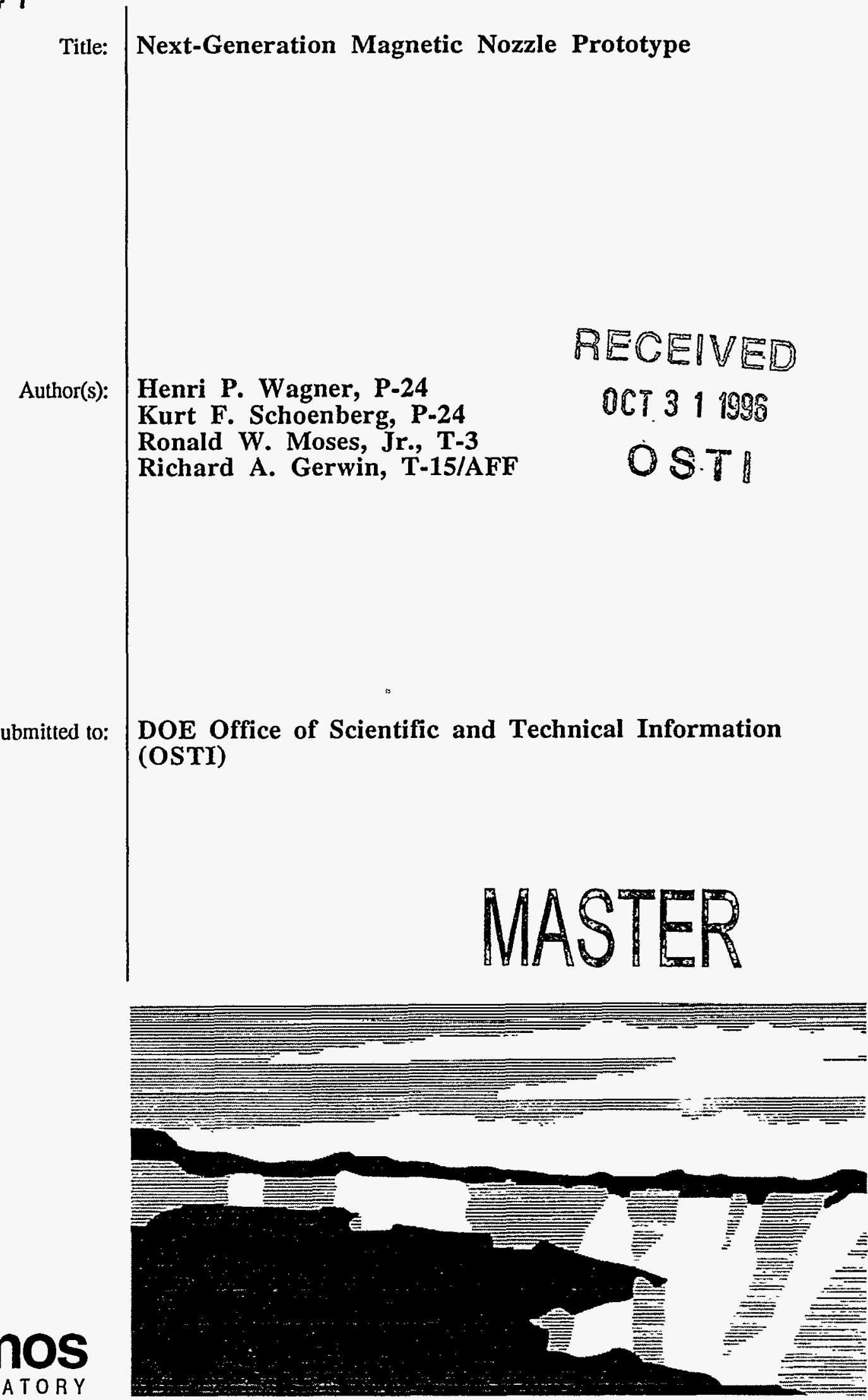

Los Alamos National Laboratory, an affirmative action/equal opportunity employer, Is operated by the University of California for the U.S. Department of Energy under contract W-7405-ENG-36. By acceptance of this article, the publisher recognizes that the U.S. Government retains a nonexclusive, royaltyfree license to pubilsh or reproduce the published form of this contribution, or to allow others to do so, for U.S. Government purposes. The Los Alamos National Laboratory requests that the publisher identify this article as work performed under the auspices of the U.S. Department of Energy. 


\section{DISCLAIMER}

This report was prepared as an account of work sponsored by an agency of the United States Government. Neither the United States Government nor any agency thereof, nor any of their employees, makes any warranty, express or implied, or assumes any legal liability or responsibility for the accuracy, completeness, or usefulness of any information, apparatus, product, or process disclosed, or represents that its use would not infringe privately owned rights. Reference herein to any specific commercial product, process, or service by trade name, trademark, manufacturer, or otherwise does not necessarily constitute or imply its endorsement, recommendation, or favoring by the United States Government or any agency thereof. The views and opinions of authors expressed herein do not necessarily state or reflect those of the United States Government or any agency thereof. 


\section{DISCLAIMER}

Portions of this document may be illegible in electronic image products. Images are produced from the best available original document. 


\title{
Next-Generation Magnetic Nozzle Prototype
}

Henri P. Wagner*, Kurt F. Schoenberg, Ronald W. Moses, Jr., and Richard. A. Gerwin

\begin{abstract}
This is the final report of a one-year, Laboratory-Directed Research and Development (LDRD) project at the Los Alamos National Laboratory (LANL). This project sought to develop a next-generation magnetic nozzle. The project engaged the fundamental physics of plasma-magnetic field interactions to attain plasma accelerator control that is significantly more advanced than the present state-of-the-art. Central to next-generation magnetic nozzle design and development is the ability to precisely predict the interaction of flowing magnetized plasma with self-generated and applied magnetic fields. This predictive capability must order physical processes in a way that preserves accuracy while allowing for the rapid evaluation of many different nozzle configurations. Large, "off-the-shelf," numerical codes are not well suited to nozzle design applications in that they lack the necessary non-ideal physics and are not well disposed to rapid design evaluation. For example, we know that both non-ideal magnetohydrodynamic effects, such as Hall drifts and finite iongyro-radius kinetics, are important constituents of magnetic nozzle performance. We built a special purpose code to allow system design.
\end{abstract}

\section{Background and Research Objectives}

Magneto-dynamic plasma acceleration has been widely studied in coaxial plasma gun devices over many decades. Their simple and robust mechanical and electrical design makes them an inexpensive and reliable technology that can provide United States industry with lowcost, high throughput, and environmentally benign manufacturing and processing capabilities, thus benefiting its international competitiveness. Possible industrial applications are pattern etching, surface engineering and treatments, cleaning and decontamination, as well as polishing

\footnotetext{
* Principal investigator, e-mail: wagner@lanl.gov
} 
and smoothing. In advanced space propulsion, such high specific impulse devices enable larger payloads to be transferred between orbits or to be carried in deep space missions.

The main objective of this project, given the strong and immediate interest of industry in plasma technology, is to develop and operate a next generation plasma processing tool, and with industry, identify new manufacturing applications. Accelerator design was guided by theoretical work and yielded scaling laws for the design of the plasma gun and its magnetic nozzle that enables precise plasma guidance from source to target. The experimental activity included the assembly of a coaxial plasma accelerator with its magnetic nozzle as designed by the modeling effort. The results will provide enhanced plasma control, strengthened capability of performance prediction and reproducibility. These are all key developmental steps for making cost-effective use of plasma accelerators in industry.

\section{Importance to LANL's Science and Technology Base and National R\&D Needs}

This project supports Laboratory core competencies in nuclear science, plasmas, and beams as well as theory, modeling, and high-performance computing. Electromagnetic plasma acceleration has strong relevance to Laboratory and national programs in advanced weapons engineering/remanufacturing applications and in advanced space propulsion. There has been a long history of plasma accelerator work at LANL with these devices being developed by John Marshall in the late 50s [1] and Joe Mather pioneering the dense plasma focus as a neutron source [2].

\section{Scientific Approach and Results to Date}

In a first step, the operational parameters of the plasma accelerator were chosen to have realistic values for industrial plasma processing. As such, the plasma gun efficiency should be above $50 \%$, the total plasma energy be around $2 \mathrm{~kJ}$, and be uniformly distributed over a 14inch diameter target surface. The average power of the system should be of the order of 10 $\mathrm{kW}$. Furthermore, a minimum particle velocity of $100 \mathrm{~km} / \mathrm{s}$ was estimated to be necessary to assure a complete energy delivery into the target surface. The voltage of the capacitor bank was limited to $30 \mathrm{kV}$ to avoid insulation problems and hard $\mathrm{x}$-ray emission in an industrial environment. Appropriate dimensions for the plasma gun were also required.

In order to make high-power-density surface processing possible and to avoid an undesired heating of the bulk material, short-pulse, high-power plasma accelerators were chosen. A simple mathematical theory describing their functioning with one circuit equation, 
two momentum equations, and an energy balance equation was readily available and easy to use for rapid designing. In a first all-parameter variation of this so-called snow-plow model, respecting the above-mentioned limits, the geometrical data of the plasma gun were determined to be 10 -inch gun length, 0.8 -inch cathode radius and 2.8-inch anode radius. A capacitor bank consisting of twelve capacitors of $1.5 \mathrm{mF}$ each, mounted on a parallel-plate power transmission line for a minimum circuit inductance, appeared to be the achievable optimum. According to the snow-plow model, this configuration provides a maximum available energy of $8.1 \mathrm{~kJ}$, of which more than half is predicted to be transferred with the plasma to the target. Once the gun geometry and the bank characteristics were chosen, the same model was used to calculate the operational window of the accelerator when the bank voltage and the gas-fill density are varied by the gun operator. This is very important information for future industrial applications of the plasma accelerator. The energy density, power density, particle velocities, and efficiencies of the energy transfer were all calculated when the bank voltage was varied between 20 and $30 \mathrm{kV}$ and the gas density was varied between $10^{-5}$ and $10^{-3} \mathrm{~kg} / \mathrm{m}^{3}$. Three-dimensional graphics have been developed to enable the designer to cover judiciously the appropriate parameters in this large phase space.

With the information obtained from the modeling described above, an engineering design of the plasma accelerator system was conducted to address all of the necessary safety and reliability concerns and regulations. Further design effort was done to assure a symmetric and reliable discharge of the bank energy into the plasma gun. From the results of previous work on plasma accelerators, this was known to be necessary to avoid serious inhomogeneities in the discharge and thus, in the energy distribution on the target. Consequently, a generally circular geometry was chosen for the system with the twelve capacitors mounted on the periphery of a double-disc flange arrangement. Furthermore, twelve spark-gap switches were added (also at the periphery) to avoid an uncontrolled gas-triggered discharge; see Figure 1. A sharp-edged ring was placed at the cathode root inside the accelerator to initiate an azimuthally homogeneous arc inside the gun. A completely new test chamber was installed to accommodate the design.

The objective of this project in modeling and theory has been to expand on the quasianalytic modeling techniques developed earlier by Wagner and Moses [3] and to apply these techniques to the design of a next generation magnetic nozzle. It has been demonstrated both in theory and in experiment that externally imposed magnetic guide fields are effective in controlling plasma deposition onto a target. In previous tests, the coils have been simple solenoids. In some cases, two or more coils have been used. The primary result of both the experimental tests and the computations was the demonstration that plasma flow tends to follow magnetic flux surfaces. Since the plasmas under consideration are not perfect conductors, there is noticeable diffusion across flux surfaces as a plasma moves from the 
accelerator to the target. Depending on the relative strength of the magnetic field and the inertia of the plasma, the magnetic surfaces are also distorted by the moving plasma.

Recognizing the effectiveness of magnetic fields for guiding plasmas and the caveats of diffusion and flux surface distortion in their usage, the next step was to respond to specific application needs. Assuming the plasma processing objective is to deliver an intense burst of energy and/or ions to a target, it is also to be expected that the plasma and energy should be delivered as uniformly as possible over a specific target surface. The plasma distribution from a coaxial source is usually most intense on axis in the target plane, or it may form a bright ring if the radius of the cathode is large compared to the target dimensions. In optical systems, the projection of an uneven intensity onto an image is known as "vignetting." To optimize use of the plasma source and produce a uniform product, the plasma should be redirected from the bright spots and enhanced in the weaker areas at the target. If one were working with light, there would be a lens or set of lenses to link the source and target. Here, such a role is played by the externally imposed magnetic field called "the magnetic nozzle."

The quasi-analytic optimization procedure works as follows. A two-dimensional computer code was written to compute the magnetic field and flux surfaces produced by any set of current loops centered on a common axis. A set of coil locations was judiciously placed around the plasma accelerator, the target and the path connecting the two. The plasma density at the muzzle of the accelerator was estimated as a function of radius and the cross-field diffusion rate was approximated. The aforementioned computer code then uses a least-squares optimization procedure to compute the current distribution at the specified coil locations that will produce the most uniform plasma flux distribution on the target. The designer may adjust the number and location of coils to improve on the target distribution and develop the best mechanical configuration of the coil set. Working with this code, coil placements were determined that provide significant improvements in compensating for plasma vignetting. This concept is deemed to be of sufficient significance to pursue patenting and will not be discussed further here.

\section{References}

1. Marshall, J., "Performance of a Hydrodynamic Plasma Gun," Physics of Fluids, 3, 134 (1960).

2. Mather, J.W., "Formation of a High Density Deuterium Plasma Focus," Physics of Fluids, 8, 366 (1965).

3. Wagner, H., R. Moses, and K. Schoenberg, "Design of a Short-Pulse Plasma Accelerator for Industrial Processes," 37th Annual APS Division of Plasma Physics Meeting, Louisville, KY, November (1995). 


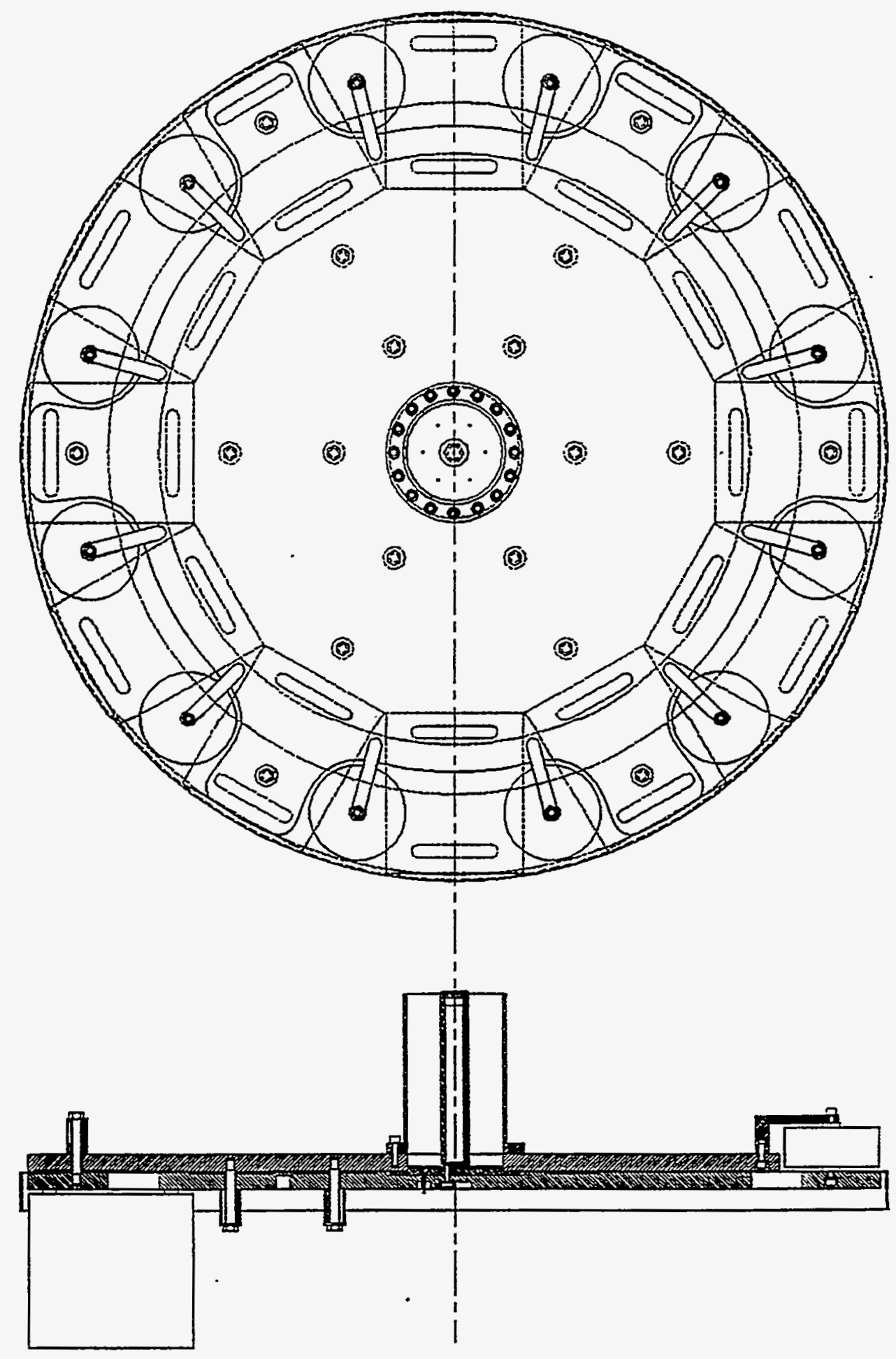

Figure 1. Assembly drawing (side and front view) of the optimized plasma accelerator. 